\title{
THE STUDY OF THE VOLATILE OILS COMPOSITION OBTAINED FROM VEGETATIVE AND GENERATIVE ORGANS OF BALLOTA NIGRA L.
}

\author{
Yana S. Kolisnyk, Alla M. Kovaleva, Olga V. Goryacha \\ National University of Pharmacy \\ Key words: black horehound; Ballota nigra; gas chromatography-mass spectrometry (GC-MS); \\ volatile oil component composition
}

\begin{abstract}
The article presents the data of the pharmacognostic study of the aerial part of black horehound (Ballota nigra L.). The aim of the work is to determine the qualitative composition and the quantitative content of the volatile components from vegetative and generative organs of black horehound. Leaves, flowers and stems of black horehound were harvested at the flowering phase in the Kharkiv region. For the first time volatile compounds of black horehound aerial parts have been investigated by GC/MS. The component composition of volatile substances in horehound herb has been studied using an Agilent Technologies $6890 \mathrm{~N}$ chromatograph with a mass spectrometry detector $5973 \mathrm{~N}$. The components have been identified according to mass spectra NIST 05 and WILEY 2007 libraries together with the programmes for identifying AMDIS and NIST. In vegetative and generative organs of horehound 48 components have been identified. Germacrene D (40.2 mg/kg), hexahydrofarnesylacetone (167.42 mg/kg), 2,6-trimethyl-4-methylene-2H-pyran (172.3 mg/kg), myristic (271 $3 \mathrm{mg} / \mathrm{kg})$, pentadecanoic $(182.1 \mathrm{mg} / \mathrm{kg})$, palmitoleic $(306.4 \mathrm{mg} / \mathrm{kg})$ and palmitic $(1620.6 \mathrm{mg} / \mathrm{kg})$ acids prevail in vegetative organs. Farnesylacetone $(68.8 \mathrm{mg} / \mathrm{kg})$, verbenone $(35.7 \mathrm{mg} / \mathrm{kg})$, myristic $(187.5 \mathrm{mg} / \mathrm{kg})$, palmitic acid $(656.5 \mathrm{mg} / \mathrm{kg})$ and palmitoleic $(196.9 \mathrm{mg} / \mathrm{kg})$ acids are the major components in generative organs. Therefore, hexahydrofarnesylacetone, palmitic and palmitoleic acids, and 2,6-trimethyl-4-methylene-2H-pyran are predominant components in the raw material studied.
\end{abstract}

Black horehound (Ballota nigra, Lamiaceae family) is a perennial herb, widely spread in Russia, Ukraine, in the countries of Western Europe, North Africa and the Caucasus [2]. The plant is not officinal, but is used in folk medicine as a sedative. In the aerial part of black horehound such diterpenoids as marrubin, ballonigrin, ballotinone, ballotenol, 7-acetoxymarrubin have been studied. The glycoside forms of phenylpropanoids verbascoside, phorsitoside, arenarioside, ballotetroside, allisonoside, lavandulifolioside, angoroside and the nonglycoside forms - $(+)-($ E)-coffeyl-L-malic acid have been found $[3,7,9,10]$. Recent studies have determined that biologically active substances (BAS) of black horehound possess nootropic, anti-oxidant, anti-arrhythmic, anti-microbial and antihypoxia activities [2, 4-6, 8]. Therefore, expedience of the comprehensive study of BAS of black horehound is an urgent question for pharmacy.

Earlier studies have shown the presence of flavonoids, tannins, iridoids, essential oil, hydroxycinnamic acid and phenol carboxylic acids in flowers (calyx, corollas), stems and leaves of black horehound [1].

The aim of this work was to study the components of essential oil of Ballota nigra by chromato-mass spectrometry.

The object of the study was samples of air-dried herb: leaves, flowers and stems of black horehound harvested at the flowering stage in the summer of 2013 in the neighbourhood of Kharkov.

\section{Materials and Methods}

The analysis of the essential oil of black horehound leaves and flowers was performed using a chromatomass spectrometer Agilent Technology HP6890 GC with a mass spectrometric detector $5973 \mathrm{~N}$.

A weighed portion of the crushed raw material $(1.0 \mathrm{~g})$ was placed in a $20 \mathrm{ml}$ "Agilent" vial, to which the internal standard (50 $\mu \mathrm{g}$ of tridecane) and $10 \mathrm{ml}$ of water were added. Volatile components of the sample were steamed for 2 hours using a reflux condenser with air cooling. Substances adsorbed on the inner surface of the reflux after cooling of the system were washed into a dry $10 \mathrm{ml}$ vial by slow adding of $3 \mathrm{ml}$ of ultrapure pentane. The washes were concentrated by blowing $(100 \mathrm{ml} / \mathrm{min})$ with ultrapure nitrogen until the residual volume of the extract was $10 \mu \mathrm{l}$, then it was completely withdrawn by a chromatographic syringe. The further concentration of the sample to the volume of $2 \mathrm{ml}$ was carried out in the syringe. The injection of the sample was performed in a splitless mode; it allowed to introduce the sample without division loss and significantly (10-20 fold) increase sensitivity of the chromatographic process. The input sample rate was $1.2 \mathrm{ml} / \mathrm{min}$ for $0.2 \mathrm{mi}-$ nutes. The chromatographic conditions were as follows: capillary chromatographic column DB-5 $(30 \mathrm{~m} \times 0.25 \mathrm{~mm})$; the carrier gas - helium, the carrier gas speed $-1.2 \mathrm{ml} / \mathrm{min}$; the thermostat temperature - from 50 to $320^{\circ} \mathrm{C}$ at the rate of $4 \% \mathrm{~min}$; the heater temperature of the sample introduction $-250^{\circ} \mathrm{C}$. From the aqueous extract vola- 
The component composition of vegetative and generative organs of black horehound

\begin{tabular}{|c|c|c|c|c|c|}
\hline $\begin{array}{l}\text { Retention } \\
\text { time, min }\end{array}$ & Compound & $\begin{array}{c}\text { Corollas, } \\
\mathrm{mg} / \mathrm{kg}\end{array}$ & $\begin{array}{l}\text { Calyx, } \\
\mathrm{mg} / \mathrm{kg}\end{array}$ & $\begin{array}{l}\text { Leaves, } \\
\mathrm{mg} / \mathrm{kg}\end{array}$ & $\begin{array}{l}\text { Stems, } \\
\mathrm{mg} / \mathrm{kg}\end{array}$ \\
\hline 5.31 & Benzaldehyde & 3.9 & - & - & 5.1 \\
\hline 6.26 & 1-Octen-3-ol & 3.5 & - & - & 10.4 \\
\hline 6.54 & 2,3,5-Trimethylpyrazine & - & - & - & 7.9 \\
\hline 6.68 & Octanal & - & 10.9 & - & - \\
\hline 7.4 & Phenylacetaldehyde & 9.3 & - & 31.3 & - \\
\hline 7.49 & Benzyl alcohol & & - & - & 9.8 \\
\hline 7.79 & Limonene & - & 10.3 & - & - \\
\hline 9.01 & Tetramethylpyrazine & - & - & - & 26.5 \\
\hline 9.17 & 2,5-Dimethyl-3-acetylfurane & 5.1 & - & - & - \\
\hline 9.69 & Nonanal & - & 21.5 & - & - \\
\hline 9.74 & Linalool & 4.9 & - & - & - \\
\hline 10.04 & 2,2,6-Trimethyl-4-methylene-2H-pyran & 172.3 & - & 14.9 & 42.5 \\
\hline 11.07 & Verbenol & - & - & - & 7.1 \\
\hline 12.34 & Methylsalicilate & 4.6 & - & - & 313 \\
\hline 12.49 & $a$-Terpineol & 4 & - & - & 6.2 \\
\hline 12.64 & Verbenone & - & - & 35.7 & 3.2 \\
\hline 12.99 & Decanal & 8.9 & 71 & 27.1 & - \\
\hline 14.59 & Isopiperitenone & - & 8.7 & - & - \\
\hline 14.84 & Ethylsalicilate & - & - & - & 7.6 \\
\hline 16.17 & 4-Vinyl-2-methoxyphenol & - & - & - & 36.1 \\
\hline 16.31 & Dodecanal & - & 519 & - & - \\
\hline 17.58 & Eugenol & 26.3 & - & 12.2 & 18.9 \\
\hline 18.76 & Caprinic acid & 39 & - & - & - \\
\hline 18.98 & Methyleugenol & 26.3 & 39.6 & 12.8 & - \\
\hline 20.04 & $\beta$-Caryophyllene & 26.8 & - & - & 2 \\
\hline 20.75 & Geranylacetone & 8.6 & 10.9 & 12.5 & 3.8 \\
\hline 21.03 & Gumulene & 11.3 & - & - & 2.2 \\
\hline 21.53 & $\beta$-Ionone-epoxyside & - & - & 36.4 & 5.4 \\
\hline 21.63 & $\beta$-Ionone & - & - & - & 3.3 \\
\hline 21.83 & Germacrene D & 40.2 & - & - & 8.2 \\
\hline 21.89 & Tetradecane & - & 6.8 & - & - \\
\hline 22.09 & Dihydroactinidiolide & - & - & 43.8 & - \\
\hline 22.94 & Pentadecane & - & 53.9 & - & - \\
\hline 23.47 & Elemicin & - & 14 & - & - \\
\hline 23.66 & Elemol & 18.5 & - & - & - \\
\hline 24.39 & Spatulenol & 15.4 & - & - & - \\
\hline 24.48 & Caryophyllene oxyde & 57.1 & - & - & 6.8 \\
\hline 24.56 & Lauric acid & - & 67.1 & - & - \\
\hline 25.19 & Benzophenone & - & 11.7 & - & - \\
\hline 26.07 & Trans-isoelemicin & - & 67.1 & - & - \\
\hline 28.6 & 11-Tetradecenic acid & - & - & - & 4.9 \\
\hline 29.05 & Miristic acid & 100.6 & 271.3 & 187.5 & 41.9 \\
\hline 29.97 & Farnesole & - & - & 32.2 & - \\
\hline 30.27 & Hexahydrofarnesylacetone & 167.4 & 60.3 & - & - \\
\hline 30.62 & Pentadecanoic acid & 50.7 & 182.1 & 121.8 & \\
\hline 31.19 & Farnesylacetone & - & - & 68.8 & - \\
\hline 31.71 & Palmitoleic acid & 44.5 & 306.4 & 196.9 & 34.7 \\
\hline 32.2 & Palmitic acid & 573.9 & 1620.6 & 656.5 & 130.9 \\
\hline
\end{tabular}


tile compounds were extracted with methylene chloride and further analyzed as described above.

Substances were identified by comparison of the mass spectra obtained with the data from mass spectra libraries NIST05 and WILEY 2007 in combination with the programmes for identification AMDIS and NIST. The content of components in the herbal drug $(\mathrm{mg} / \mathrm{kg})$ is given in Table.

\section{Results and Discussion}

Qualitative and quantitative compositions of essential oils of various organs of horehound are given in $\mathrm{mg} /$ $\mathrm{kg}$ (Table). As the result of the research 48 components of essential oil have been identified and quantified in generative and vegetative organs. Quantitatively sesquiterpenoids and substances of different origin (aldehydes, hydrocarbons, and fatty acids) are predominant, in smaller concentration monoterpenoids and aromatic compounds have been found. Germacrene D $(40.2 \mathrm{mg} / \mathrm{kg})$, hexahydrofarnesylacetone $(167.42 \mathrm{mg} / \mathrm{kg}), 2,6$-trimethyl-4-methylene-2H-pyran (172.3 $\mathrm{mg} / \mathrm{kg})$, myristic $(271.3 \mathrm{mg} / \mathrm{kg})$, pentadecanoic $(182.1 \mathrm{mg} / \mathrm{kg})$, palmitoleic $(306.4 \mathrm{mg} / \mathrm{kg})$ and palmitic $(1620.6 \mathrm{mg} / \mathrm{kg})$ acids prevail in vegetative organs. Farnesylacetone $(68.8 \mathrm{mg} / \mathrm{kg})$, verbenone $(35.7 \mathrm{mg} / \mathrm{kg})$, myristic $(187.5 \mathrm{mg} / \mathrm{kg})$, palmitic acid $(656.5 \mathrm{mg} / \mathrm{kg})$ and palmitoleic $(196.9 \mathrm{mg} / \mathrm{kg})$ acids are the major components in generative organs.

\section{CONCLUSIONS}

1. The Chromato-mass spectrometric research of volatile substances of vegetative and generative organs of black horehound has been carried out; 48 components have been identified and quantified.

2. The predominant components in the raw material studied are hexahydrofarnesylacetone, palmitic acid, palmitoleic acid and 2,6-trimethyl-4-methylene-2H-pyran.

3. The results obtained will be used for further phytochemical research of these herbal drugs.

\section{REFERENCES}

1. Ковалева А.М., Колесник Я.С., Седова А.Б. // Вестник Пермской государственной фармачевтической академии. Современные тенденции и перспективы развития фармацевтического образования и науки в России и за рубежсм: Матер. научно-практ. конф. с междунар. участием (21-23 ноября 2013 года). - 2013. - №11. - C. 76-78.

2. Круглая А.А., Тираспольская С.Г., Алфимова Г.В. и др. // Известия Самарского научного иентра РАН. 2012. - T. 14, №5(3). - C. 726-730.

3. Bertrand M., Tillequin F., Bailleul F. // Biochemical Systematics and Ecol. - 2001. - Vol. 28 (10). - P. 1031-1033.

4. Çitoğlu G., Coban T., Sever B. et al. // J. of Ethnopharmacol. - 2004. - Vol. 92 (2-3). - P. 275-280.

5. Çitoğlu G., Özbek H., Sever B. // Eastern J. of Medicine. - 2005. - Vol. 10. - P. 24-28.

6. Dulger B., Sener A. // African J. of Microbiol. Res. - 2010. - Vol. 4 (12). - P. 1235-1238.

7. Enikö T., Gábor J., Imre M. et al. // J. of Planar Chromatography-Modern TLC. - 2009. - Vol. 22 (4). P. 293-296.

8. Ezer N., Şahin F., Toker M. // Israel J. of Plant Sci. - 1999. - Vol. 47 (1). - P. 43-48.

9. Sahpaz S., Skaltsounis A.L., Bailleul F. // Biochem. Syst. Ecol. - 2002. - Vol. 30. - P. 601-604.

10. Sever B. The investigation of diterpenoid and flavonoid contents of Ballota species growing in Turkey. $-P h D$ Thesis. - Ankara, 2002. - 134 p.

\section{ДОСЛІДЖЕННЯ СКЛАДУ ЕФІРНОЇ ОЛІЇ ВЕГЕТАТИВНИХ І ГЕНЕРАТИВНИХ ОРГАНІВ \\ BALLOTA NIGRA L. \\ Я.С.Колісник, А.М.Ковальова, О.В.Горяча \\ Ключові слова: М'яточник чорний; Ballota nigra L.; хромато-мас-спектрометрія; \\ компонентний склад ефрірної олії}

Наведені дані фрармакогностичного вивчення надземної частини м'яточника чорного (Ballota nigra L.). Метою роботи є визначення якісного складу та кількісного вмісту летких компонентів вегетативних та генеративних органів м'яточника чорного. В якості сировини використовували листя, квітки і стебла, заготовлені в фразу цвітіння в Харківській області. Вперше методом хромато-мас-спектрометрії проведено дослідження летких речовин надземної частини м'яточника чорного. У вегетативних та генеративних органах м'яточника ідентифріковано 48 компонентів. У вегетативних органах переважають гермакрен D (40,2 мг/кг), гексагідрофрарнезилацетон (167,42 мг/к2), 2,6-триметил-4-метилен-2Н-піран (172,3 мә/кг), міристинова (271,3 мг/к2), пентадеканова (182,1 мг/к2), пальмітолеїнова (306,4 мг/к2) і пальмітинова кислоти (1620,6 мг/ка). У генеративних органах рослини переважають фрарнезилацетон (68,8 мг/к2), вербенон (35,7 мг/к2), міристинова (187,5 мг/к2), пальмітинова (656,5 мг/к2) і пальмітолеїнова (196, 9 мг/кг) кислоти. Домінуючими компонентами у досліджуваній сировині є гексагідрофрарнезилацетон, пальмітинова та пальмітолеїнова кислоти, а також 2,6-триметил-4-метилен-2Н-піран. 


\section{ИССЛЕДОВАНИЕ СОСТАВА ЭФИРНОГО МАСЛА ВЕГЕТАТИВНЫХ И ГЕНЕРАТИВНЫХ OPГAHOB BALLOTA NIGRA L. \\ Я.С.Колесник, А.М.Ковалева, О.В.Горячая}

Ключевые слова: Белокудренник черный; Ballota nigra L.; хромато-масс-спектрометрия; компонентный состав эфрирного масла

Приведены данные фрармакогностического изучения надземной части белокудренника черного (Ballota nigra L.). Целью работы было определение качественного состава и количественного содержания летучих компонентов вегетативных и генеративных органов белокудренника черного. В качестве сырья использовали листья, иветки и стебли, заготовленные в фразу цветения в Харьковской области. Впервые методом хромато-масс-спектрометрии проведено исследование летучих веществ надземной части белокудренника черного. Компонентный состав летучих веществ травы белокудренника исследовали на хроматограdpe Agilent Technologies 6890N с масс-спектрометрическим детектором 5973N. Компоненты идентифрицировали с помощью библиотеки масс-спектров NIST 05 и WILEY 2007 совместно с программами для идентификации AMDIS и NIST. В вегетативных и генеративных органах белокудренника идентифицировано 48 компонентов. В вегетативных органах преобладают гермакрен D (40,2 мг/к2), гексагидрофрарнезилацетон (167,42 мг/к2), 2,6-триметил4-метилен-2Н-пиран (172,3 мг/к2), миристиновая (271,3 мг/к2), пентадекановая (182, 1 мг/к2), пальмитолеиновая (306,4 мг/ка) и пальмитиновая кислоты (1620,6 м2/ка). В генеративных органах растения преобладают фрарнезилацетон (68,8 мг/к2), вербенон (35,7 мг/к2), миристиновая (187,5 мг/ка), пальмитиновая (656,5 мг/к2) и пальмитолеиновая (196, 9 мг/кг) кислоты. Доминирующими компонентами в исследуемом сырье являются гексагидрофрарнезилацетон, пальмитиновая и пальмитолеиновая кислоты, а также 2, 6-триметил-4-метилен$2 \mathrm{H}$-пиран. 